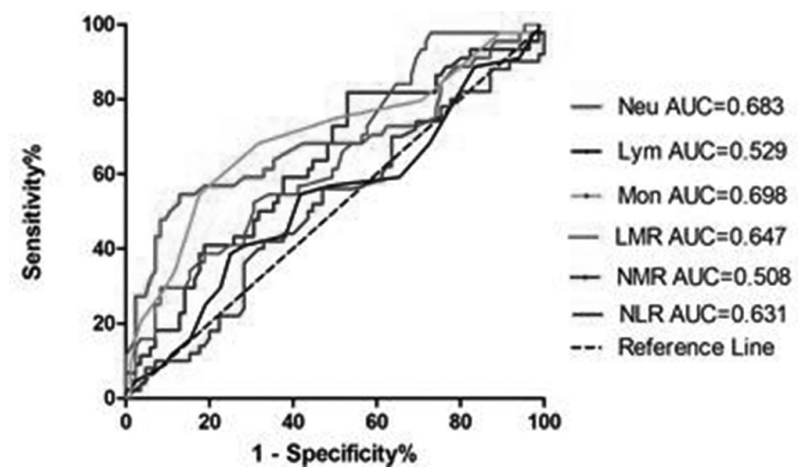

Abstract IDDF2019-ABS-0186 Figure 2 Biomarker performance. Receiver operator curves of the various leukocyte subtypes and ratios in $\mathrm{CD}$ active compared with $\mathrm{CD}$ remission

Conclusions A low LMR represents an inexpensive, readily available test with a promising value to identify disease activity in UC patients. Whereas none of the inflammatory biomarkers showed a discriminative value in disease activity of CD.

\section{IDDF2019-ABS-0189 YOGA-ENHANCED COGNITIVE BEHAVIOURAL THERAPY (Y-CBT) VERSUS RIFAMIXIN-PROBIOTIC SEQUENTIAL TREATMENT FOR IRRITABLE BOWEL SYNDROME (IBS): A RANDOMISED CLINICAL TRIAL}

${ }^{1}$ Debanjan Banerjee*, ${ }^{2}$ Sujata Bhatt, ${ }^{1}$ Soumalyo Chatterjee, ${ }^{3}$ Rajesh Sharma. ${ }^{1}$ National Institute of Mental Health and Neurosciences (NIMHANS), Bangalore, India; ${ }^{2}$ St. Johns Medical College, Bangalore, India; ${ }^{3}$ Bangalore Institute of Gastroenterology, India

\subsection{6/gutjnl-2019-IDDFabstracts. 188}

Background Irritable bowel syndrome (IBS) is a common and disabling gastrointestinal disorder. It adds to immense morbidity. Recent theories implicate visceral hypersensitivity, stress and psycho-social factors in the causation of IBS. Kundalini Yoga affects the body homeostatis and cognitive behavioural therapy (CBT) when used in conjunction, reverse stressinduced underactivity of the parasympathetic nervous system. Rifamixin (a poorly absorbed antibiotic) and probiotics are common treatments of IBS used sequentially, altering gut flora. The primary objective here was to examine the effect of Yoga-enhanced CBT (Y-CBT) versus Rifamixin-probiotic treatment on patients with IBS.

Methods Sixty patients diagnosed as IBS entered a single-blind randomised controlled trial involving Y-CBT or rifamixin-probiotic sequential treatment for 12 weeks. The study was approved by the Institute Ethics Board. The Y-CBT group had $\underline{2}$ weekly sessions of (Kundalini Yoga + CBT) by trained professionals while the other group was treated with tablet Rifamixin $550 \mathrm{mg}$ three times a day for $\underline{6}$ weeks followed by a probiotic capsule (containing B. infantis and L. rhamnosus) daily for next $\underline{6}$ weeks. Both groups received a total of three sessions of Mediterranean diet counselling for uniformity. The primary outcome was changed in gastronintestinal symptoms (IBS-SSS). Secondary measures were quality of life (IBS-QOL), depression (HAM-D), anxiety (HAM-A), perceived health status (PHSQ) and safety of interventions. Outcomes were assessed at 12 and 24 weeks, being blinded to allocation.
Results NO statistically significant difference was found between intervention groups with regard to IBS-SSS score at either $12(\mathrm{Diff}=34.2,95 \% \mathrm{CI}=-12.9,76.6, \mathrm{P}=0.121)$ or 24 weeks $\quad(\mathrm{Diff}=32.9,95 \% \mathrm{CI}=-5.31,70.14, \mathrm{P}=0.071)$. Within group comparisons showed statistically significant effect for both the interventions. However, the Y-CBT group fared better in quality of life, anxiety and perceived health status. Drop-outs were more in the rifamixin-probiotic group $(\mathrm{P}=0.9)$. No serious adverse effects were reported.

Conclusions Yoga, being a traditional and simple intervention when used with CBT, can be as effective as the commonly used medicines in IBS. More research is warranted about their combined use.

\section{IDDF2019-ABS-0191 INITIAL EXPERIENCE OF FAECAL MICROBIOTA TRANSPLANTATION WITH FROZEN STOOLS FOR THE TREATMENT OF RECURRENT OR REFRACTORY CLOSTRIDIOIDES DIFFICILE INFECTION - A RETROSPECTIVE REVIEW FROM A QUATERNARY REFERRAL CENTRE IN HONG KONG}

${ }^{1}$ Rashid Lui*, 'Louis Lau, 'Ting-ting Chan, 'Sunny Wong, ${ }^{1}$ Kitty Cheung, 'Amy Li,

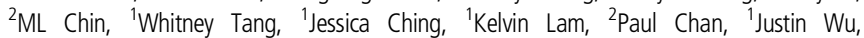
${ }^{1} J o s e p h$ Sung, 'Francis Chan, 'Siew CNG. 'Institute of Digestive Disease, The Chinese University of Hong Kong, Hong Kong; ${ }^{2}$ Department of Microbiology, The Chinese University of Hong Kong, Hong Kong

\subsection{6/gutjnl-2019-IDDFabstracts. 189}

Background Clostridioides difficile infection (CDI) is a major cause of nosocomial infection leading to significant morbidity and mortality. Faecal microbiota transplantation (FMT) has emerged as a promising option for recurrent and refractory CDI. This study aims to assess the safety, efficacy and feasibility of FMT using frozen stools from a newly established stool biobank for the treatment of CDI in Hong Kong.

Methods We conducted a single-centre, retrospective study for all consecutive cases of recurrent/refractory CDI who had FMT with frozen stools performed from 2017 to present. Clinical demographics, outcome, and safety parameters were collected from the Clinical Management System of the Hospital Authority, Hong Kong or from the review of case notes.

Results A total of 19 patients with recurrent or refractory CDI and FMT performed using frozen stools from the CUHK stool biobank were identified [median age 70 years (interquartile range of 58.5 - 77.5 years); $68.4 \%$ males]. Almost half of the cases were in a bedridden or chair-bound state, a surrogate of a poor functional status. The majority $(>80 \%)$ of patients were hospitalized within the recent $\underline{3}$ months or were long-term care facility residents. FMT was delivered via feeding tube in 1 (5.3\%), esophagogastroduodenoscopy (OGD) in $12(63.2 \%)$, or colonoscopy in $6(31.6 \%)$ of the patients. Resolution of diarrhoea without relapse within 8 weeks was achieved in 16 out of 19 patients (84.2\%). The procedure was generally well tolerated with no serious adverse events attributable to FMT. The most common complication after FMT was abdominal pain $(\mathrm{n}=3,15.8 \%)$. Bloating was reported in 1 patient $(5.3 \%)$. These results suggest that the cure rates of FMT using frozen stools for recurrent or refractory CDI were comparable with those reported in the literature with an excellent safety profile. 